Research Article

\title{
Convergence Theorems Concerning Hybrid Methods for Strict Pseudocontractions and Systems of Equilibrium Problems
}

\author{
Peichao Duan \\ College of Science, Civil Aviation University of China, Tianjin 300300, China \\ Correspondence should be addressed to Peichao Duan, pcduancauc@126.com \\ Received 23 May 2010; Revised 23 August 2010; Accepted 26 August 2010 \\ Academic Editor: S. Reich
}

Copyright (C) 2010 Peichao Duan. This is an open access article distributed under the Creative Commons Attribution License, which permits unrestricted use, distribution, and reproduction in any medium, provided the original work is properly cited.

Let $\left\{S_{i}\right\}_{i=1}^{N}$ be $N$ strict pseudo-contractions defined on a closed and convex subset $C$ of a real Hilbert space $H$. We consider the problem of finding a common element of fixed point set of these mappings and the solution set of a system of equilibrium problems by parallel and cyclic algorithms. In this paper, new iterative schemes are proposed for solving this problem. Furthermore, we prove that these schemes converge strongly by hybrid methods. The results presented in this paper improve and extend some well-known results in the literature.

\section{Introduction}

Let $H$ be a real Hilbert space with inner product $\langle\cdot, \cdot\rangle$ and norm $\|\cdot\|$. Let $C$ be a nonempty, closed, and convex subset of $H$.

Let $\left\{F_{k}\right\}$ be a countable family of bifunctions from $C \times C$ to $\mathbb{R}$, where $\mathbb{R}$ is the set of real numbers. Combettes and Hirstoaga [1] considered the following system of equilibrium problems:

$$
\text { finding } x \in C \text { such that } F_{k}(x, y) \geq 0, \quad \forall k \in \Gamma, \forall y \in C \text {, }
$$

where $\Gamma$ is an arbitrary index set. If $\Gamma$ is a singleton, then problem (1.1) becomes the following equilibrium problem:

$$
\text { finding } x \in C \text { such that } F(x, y) \geq 0, \quad \forall y \in C \text {. }
$$

The solution set of (1.2) is denoted by $\mathrm{EP}(F)$. 
A mapping $S$ of $C$ is said to be a $\mathcal{\kappa}$-strict pseudocontraction if there exists a constant $\kappa \in[0,1)$ such that

$$
\|S x-S y\|^{2} \leq\|x-y\|^{2}+\kappa\|(I-S) x-(I-S) y\|^{2}
$$

for all $x, y \in C$; see [2]. We denote the fixed point set of $S$ by $F(S)$, that is, $F(S)=\{x \in$ $C: S x=x\}$.

Note that the class of strict pseudocontractions properly includes the class of nonexpansive mappings which are mapping $S$ on $C$ such that

$$
\|S x-S y\| \leq\|x-y\|
$$

for all $x, y \in C$. That is, $S$ is nonexpansive if and only if $S$ is a 0 -strict pseudocontraction.

The problem (1.1) is very general in the sense that it includes, as special cases, optimization problems, variational inequalities, minimax problems, Nash equilibrium problem in noncooperative games, and others; see, for instance, $[1,3,4]$ and the references therein. Some methods have been proposed to solve the equilibrium problem (1.1); related work can also be found in [5-8].

Recently, Acedo and Xu [9] considered the problem of finding a common fixed point of a finite family of strict pseudo-contractive mappings by the parallel and cyclic algorithms. Very recently, Duan and Zhao [10] considered new hybrid methods for equilibrium problems and strict pseudocontractions. In this paper, motivated by [5, 8-12], applying parallel and cyclic algorithms, we obtain strong convergence theorems for finding a common element of the fixed point set of a finite family of strict pseudocontractions and the solution set of the system of equilibrium problems (1.1) by the hybrid methods.

We will use the following notations:

$(1) \rightarrow$ for the weak convergence and $\rightarrow$ for the strong convergence,

(2) $\omega_{w}\left(x_{n}\right)=\left\{x: \exists x_{n_{j}} \rightarrow x\right\}$ denotes the weak $\omega$-limit set of $\left\{x_{n}\right\}$.

\section{Preliminaries}

We will use the facts and tools in a real Hilbert space $H$ which are listed below.

Lemma 2.1. Let $H$ be a real Hilbert space. Then the following identities hold:

(i) $\|x-y\|^{2}=\|x\|^{2}-\|y\|^{2}-2\langle x-y, y\rangle$, for all $x, y \in H$,

(ii) $\|t x+(1-t) y\|^{2}=t\|x\|^{2}+(1-t)\|y\|^{2}-t(1-t)\|x-y\|^{2}$, for all $t \in[0,1]$, for all $x, y \in H$.

Lemma 2.2 (see [6]). Let $H$ be a real Hilbert space. Given a nonempty, closed, and convex subset $C \subset H$, points $x, y, z \in H$, and a real number $a \in \mathbb{R}$, then the set

$$
\left\{v \in C:\|y-v\|^{2} \leq\|x-v\|^{2}+\langle z, v\rangle+a\right\}
$$

is convex (and closed). 
Recall that given a nonempty, closed, and convex subset $C$ of a real Hilbert space $H$, for any $x \in$, there exists the unique nearest point in $C$, denoted by $P_{C} x$, such that

$$
\left\|x-P_{C} x\right\| \leq\|x-y\|
$$

for all $y \in C$. Such a $P_{C}$ is called the metric (or the nearest point) projection of $H$ onto $C$. As we all know $y=P_{C} x$ if and only if there holds the relation

$$
\langle x-y, y-z\rangle \geq 0 \quad \forall z \in C
$$

Lemma 2.3 (see [13]). Let $C$ be a nonempty, closed, and convex subset of $H$. Let $\left\{x_{n}\right\}$ be a sequence in $H$ and $u \in H$. Let $q=P_{C} u$. Suppose that $\left\{x_{n}\right\}$ is such that $\omega_{w}\left(x_{n}\right) \subset C$ and satisfies the following condition:

$$
\left\|x_{n}-u\right\| \leq\|u-q\| \quad \forall n .
$$

Then $x_{n} \rightarrow q$.

Proposition 2.4 (see [9]). Let C be a nonempty, closed, and convex subset of a real Hilbert space $H$.

(i) If $T: C \rightarrow C$ is a $\mathcal{\kappa}$-strict pseudocontraction, then $T$ satisfies the Lipschitz condition

$$
\|T x-T y\| \leq \frac{1+\kappa}{1-\kappa}\|x-y\|, \quad \forall x, y \in C
$$

(ii) If $T: C \rightarrow C$ is a $\kappa$-strict pseudocontraction, then the mapping $I-T$ is demiclosed (at 0 ). That is, if $\left\{x_{n}\right\}$ is a sequence in $C$ such that $x_{n} \rightarrow x$ and $(I-T) x_{n} \rightarrow 0$, then $(I-T) x=0$.

(iii) If $T: C \rightarrow C$ is a $\kappa$-strict pseudocontraction, then the fixed point set $F(T)$ of $T$ is closed and convex. Therefore the projection $P_{F(T)}$ is well defined.

(iv) Given an integer $N \geq 1$, assume that, for each $1 \leq i \leq N, T_{i}: C \rightarrow C$ is a $\kappa_{i}$-strict pseudocontraction for some $0 \leq \kappa_{i}<1$. Assume that $\left\{\lambda_{i}\right\}_{i=1}^{N}$ is a positive sequence such that $\sum_{i=1}^{N} \lambda_{i}=1$. Then $\sum_{i=1}^{N} \lambda_{i} T_{i}$ is a $\mathcal{\kappa}$-strict pseudocontraction, with $\kappa=\max \left\{\kappa_{i}: 1 \leq i \leq\right.$ $N\}$.

(v) Let $\left\{T_{i}\right\}_{i=1}^{N}$ and $\left\{\lambda_{i}\right\}_{i=1}^{N}$ be given as in item (iv). Suppose that $\left\{T_{i}\right\}_{i=1}^{N}$ has a common fixed point. Then

$$
F\left(\sum_{i=1}^{N} \lambda_{i} T_{i}\right)=\bigcap_{i=1}^{N} F\left(T_{i}\right) .
$$

Lemma 2.5 (see [2]). Let $S: C \rightarrow H$ be a $\boldsymbol{\kappa}$-strict pseudocontraction. Define $T: C \rightarrow H$ by $T x=\lambda x+(1-\lambda) S x$ for any $x \in C$. Then, for any $\lambda \in[\kappa, 1), T$ is a nonexpansive mapping with $F(T)=F(S)$. 

conditions:

For solving the equilibrium problem, let one assume that the bifunction F satisfies the following

(A1) $F(x, x)=0$ for all $x \in C$;

(A2) $F$ is monotone, that is, $F(x, y)+F(y, x) \leq 0$ for any $x, y \in C$;

(A3) for each $x, y, z \in C$, limsup $\sup _{t \rightarrow 0} F(t z+(1-t) x, y) \leq F(x, y)$;

(A4) $F(x, \cdot)$ is convex and lower semicontinuous for each $x \in C$.

Lemma 2.6 (see [3]). Let $C$ be a nonempty, closed, and convex subset of $H$, let $F$ be bifunction from $C \times C$ to $\mathbb{R}$ which satisfies conditions (A1)-(A4), and let $r>0$ and $x \in H$. Then there exists $z \in C$ such that

$$
F(z, y)+\frac{1}{r}\langle y-z, z-x\rangle \geq 0, \quad \forall y \in C
$$

Lemma 2.7 (see [1]). For $r>0, x \in H$, define the mapping $T_{r}: H \rightarrow C$ as follows:

$$
T_{r}(x)=\{z \in C \mid F(z, y)+(1 / r)\langle y-z, z-x\rangle \geq 0, \forall y \in C\}
$$

for all $x \in H$. Then, the following statements hold:

(i) $T_{r}$ is single valued;

(ii) $T_{r}$ is firmly nonexpansive, that is, for any $x, y \in H$,

$$
\left\|T_{r} x-T_{r} y\right\|^{2} \leq\left\langle T_{r} x-T_{r} y, x-y\right\rangle
$$

(iii) $F\left(T_{r}\right)=\mathrm{EP}(F)$;

(iv) $\mathrm{EP}(F)$ is closed and convex.

\section{Parallel Algorithm}

In this section, we apply the hybrid methods to the parallel algorithm for finding a common element of the fixed point set of strict pseudocontractions and the solution set of the problem (1.1) in Hilbert spaces.

Theorem 3.1. Let $C$ be a nonempty, closed, and convex subset of a real Hilbert space $H$, and let $F_{k}, k \in\{1,2, \ldots, M\}$, be bifunctions from $C \times C$ to $\mathbb{R}$ which satisfies conditions (A1)-(A4). Let, for each $1 \leq i \leq N, S_{i}: C \rightarrow C$ be a $\kappa_{i}$-strict pseudocontraction for some $0 \leq \kappa_{i}<1$. Let $\kappa=$ $\max \left\{\kappa_{i}: 1 \leq i \leq N\right\}$. Assume that $\Omega=\cap_{i=1}^{N} F\left(S_{i}\right) \cap\left(\cap_{k=1}^{M} \operatorname{EP}\left(F_{k}\right)\right) \neq \emptyset$. Assume also that $\left\{\eta_{i}^{(n)}\right\}_{i=1}^{N}$ is a finite sequence of positive numbers such that $\sum_{i=1}^{N} \eta_{i}^{(n)}=1$ for all $n \in \mathbb{N}$ and $\inf _{n \geq 1} \eta_{i}^{(n)}>0$ for all $1 \leq i \leq N$. Let the mapping $A_{n}$ be defined by

$$
A_{n}=\sum_{i=1}^{N} \eta_{i}^{(n)} S_{i}
$$


Given $x_{1} \in C$, let $\left\{x_{n}\right\},\left\{u_{n}\right\}$, and $\left\{y_{n}\right\}$, be sequences which are generated by the following algorithm:

$$
\begin{aligned}
u_{n} & =T_{r_{M, n}}^{F_{M}} T_{r_{M-1, n}}^{F_{M-1}} \cdots T_{r_{2, n}}^{F_{2}} T_{r_{1, n}}^{F_{1}} x_{n}, \\
A_{n}^{\lambda_{n}} & =\lambda_{n} I+\left(1-\lambda_{n}\right) A_{n}, \\
y_{n} & =\alpha_{n} x_{n}+\left(1-\alpha_{n}\right) A_{n}^{\lambda_{n}} u_{n}, \\
C_{n} & =\left\{z \in C:\left\|y_{n}-z\right\| \leq\left\|x_{n}-z\right\|\right\}, \\
Q_{n} & =\left\{z \in C:\left\langle x_{n}-z, x_{1}-x_{n}\right\rangle \geq 0\right\}, \\
x_{n+1} & =P_{C_{n} \cap Q_{n}} x_{1},
\end{aligned}
$$

where $\left\{\alpha_{n}\right\} \subset[0, a]$ for some $a \in[0,1),\left\{\lambda_{n}\right\} \subset[\kappa, b]$ for some $b \in[\kappa, 1)$, and $\left\{r_{k, n}\right\} \subset(0, \infty)$ satisfies $\lim \inf _{n \rightarrow \infty} r_{k, n}>0$ for all $k \in\{1,2, \ldots, M\}$. Then, $\left\{x_{n}\right\}$ converge strongly to $P_{\Omega} x_{1}$.

Proof. Denote $\Theta_{n}^{k}=T_{r_{k, n}}^{F_{k}} \cdots T_{r_{2, n}}^{F_{2}} T_{r_{1, n}}^{F_{1}}$ for every $k \in\{1,2, \ldots, M\}$ and $\Theta_{n}^{0}=I$ for all $n \in \mathbb{N}$. Therefore $u_{n}=\Theta_{n}^{M} \mathrm{x}_{n}$. The proof is divided into six steps.

Step 1 . The sequence $\left\{x_{n}\right\}$ is well defined.

It is obvious that $C_{n}$ is closed and $Q_{n}$ is closed and convex for every $n \in \mathbb{N}$. From Lemma 2.2, we also get that $C_{n}$ is convex.

Take $p \in \Omega$, since for each $k \in\{1,2, \ldots, M\}, T_{r_{k, n}}^{F_{k}}$ is nonexpansive, $p=T_{r_{k, n}}^{F_{k}} p$, and $u_{n}=\Theta_{n}^{M} x_{n}$, we have

$$
\left\|u_{n}-p\right\|=\left\|\Theta_{n}^{M} x_{n}-\Theta_{n}^{M} p\right\| \leq\left\|x_{n}-p\right\|
$$

for all $n \in \mathbb{N}$. From Proposition 2.4, Lemma 2.5, and (3.3), we get

$$
\left\|y_{n}-p\right\|=\left\|\alpha_{n} x_{n}+\left(1-\alpha_{n}\right) A_{n}^{\lambda_{n}} u_{n}-p\right\| \leq \alpha_{n}\left\|x_{n}-p\right\|+\left(1-\alpha_{n}\right)\left\|A_{n}^{\lambda_{n}} u_{n}-p\right\| \leq\left\|x_{n}-p\right\| .
$$

So $p \in C_{n}$ for all $n \in \mathbb{N}$. Thus $\Omega \subset C_{n}$. Next we will show by induction that $\Omega \subset Q_{n}$ for all $n \in \mathbb{N}$. For $n=1$, we have $\Omega \subset C=Q_{1}$. Assume that $\Omega \subset Q_{n}$ for some $n \geq 1$. Since $x_{n+1}=P_{C_{n} \cap Q_{n}} x_{1}$, we obtain

$$
\left\langle x_{n+1}-z, x_{1}-x_{n+1}\right\rangle \geq 0, \quad \forall z \in C_{n} \cap Q_{n} .
$$

As $\Omega \subset C_{n} \cap Q_{n}$ by induction assumption, the inequality holds, in particular, for all $z \in \Omega$. This together with the definition of $Q_{n+1}$ implies that $\Omega \subset Q_{n+1}$. Hence $\Omega \subset Q_{n}$ holds for all $n \geq 1$. Thus $\Omega \subset C_{n} \cap Q_{n}$, and therefore the sequence $\left\{x_{n}\right\}$ is well defined.

Step 2.

$$
\text { If } q=P_{\Omega} x_{1}, \quad \text { then }\left\|x_{n}-x_{1}\right\| \leq\left\|x_{1}-q\right\| \text {. }
$$


From the definition of $Q_{n}$ we imply that $x_{n}=P_{Q_{n}} x_{1}$. This together with the fact that $\Omega \subset Q_{n}$ further implies that

$$
\left\|x_{n}-x_{1}\right\| \leq\left\|x_{1}-p\right\| \quad \forall p \in \Omega
$$

Then $\left\{x_{n}\right\}$ is bounded and (3.6) holds. From (3.3), (3.4), and Proposition 2.4 (i), we also obtain that $\left\{u_{n}\right\},\left\{y_{n}\right\}$, and $\left\{S_{i} x_{n}\right\}$ are bounded.

Step 3. The following limit holds:

$$
\lim _{n \rightarrow \infty}\left\|x_{n+1}-x_{n}\right\|=0
$$

From $x_{n}=P_{Q_{n}} x_{1}$ and $x_{n+1} \in Q_{n}$, we get $\left\langle x_{n+1}-x_{n}, x_{n}-x_{1}\right\rangle \geq 0$. This together with Lemma 2.1 (i) implies that

$$
\begin{aligned}
\left\|x_{n+1}-x_{n}\right\|^{2} & =\left\|x_{n+1}-x_{1}-\left(x_{n}-x_{1}\right)\right\|^{2} \\
& =\left\|x_{n+1}-x_{1}\right\|^{2}-\left\|x_{n}-x_{1}\right\|^{2}-2\left\langle x_{n+1}-x_{n}, x_{n}-x_{1}\right\rangle \\
& \leq\left\|x_{n+1}-x_{1}\right\|^{2}-\left\|x_{n}-x_{1}\right\|^{2} .
\end{aligned}
$$

Then $\left\|x_{n}-x_{1}\right\| \leq\left\|x_{n+1}-x_{1}\right\|$, that is, the sequence $\left\{\left\|x_{n}-x_{1}\right\|\right\}$ is nondecreasing. Since $\left\{\left\|x_{n}-x_{1}\right\|\right\}$ is bounded, $\lim _{n \rightarrow \infty}\left\|x_{n}-x_{1}\right\|$ exists. Then (3.8) holds.

Step 4. The following limit holds:

$$
\lim _{n \rightarrow \infty}\left\|A_{n} x_{n}-x_{n}\right\|=0
$$

From $x_{n+1} \in C_{n}$, we have

$$
\left\|y_{n}-x_{n}\right\| \leq\left\|x_{n+1}-x_{n}\right\|+\left\|y_{n}-x_{n+1}\right\| \leq 2\left\|x_{n+1}-x_{n}\right\|
$$

By (3.6), we obtain

$$
\lim _{n \rightarrow \infty}\left\|y_{n}-x_{n}\right\|=0
$$

Next we will show that

$$
\lim _{n \rightarrow \infty}\left\|\Theta_{n}^{k} x_{n}-\Theta_{n}^{k-1} x_{n}\right\|=0, \quad k=1,2, \ldots, M .
$$


Journal of Inequalities and Applications

Indeed, for $p \in \Omega$, it follows from the firm nonexpansivity of $T_{r_{k, n}}^{F_{k}}$ that for each $k \in$ $\{1,2, \ldots, M\}$, we have

$$
\begin{aligned}
\left\|\Theta_{n}^{k} x_{n}-p\right\|^{2} & =\left\|T_{r_{k, n}}^{F_{k}} \Theta_{n}^{k-1} x_{n}-T_{r_{k, n}}^{F_{k}} p\right\|^{2} \\
& \leq\left\langle\Theta_{n}^{k} x_{n}-p, \Theta_{n}^{k-1} x_{n}-p\right\rangle \\
& =\frac{1}{2}\left(\left\|\Theta_{n}^{k} x_{n}-p\right\|^{2}+\left\|\Theta_{n}^{k-1} x_{n}-p\right\|^{2}-\left\|\Theta_{n}^{k} x_{n}-\Theta_{n}^{k-1} x_{n}\right\|^{2}\right) .
\end{aligned}
$$

Thus we get

$$
\left\|\Theta_{n}^{k} x_{n}-p\right\|^{2} \leq\left\|\Theta_{n}^{k-1} x_{n}-p\right\|^{2}-\left\|\Theta_{n}^{k} x_{n}-\Theta_{n}^{k-1} x_{n}\right\|^{2}, \quad k=1,2, \ldots, M,
$$

which implies that, for each $k \in\{1,2, \ldots, M\}$,

$$
\begin{aligned}
\left\|\Theta_{n}^{k} x_{n}-p\right\|^{2} \leq & \left\|\Theta_{n}^{0} x_{n}-p\right\|^{2}-\left\|\Theta_{n}^{k} x_{n}-\Theta_{n}^{k-1} x_{n}\right\|^{2}-\left\|\Theta_{n}^{k-1} x_{n}-\Theta_{n}^{k-2} x_{n}\right\|^{2} \\
& -\cdots-\left\|\Theta_{n}^{2} x_{n}-\Theta_{n}^{1} x_{n}\right\|^{2}-\left\|\Theta_{n}^{1} x_{n}-\Theta_{n}^{0} x_{n}\right\|^{2} \\
\leq & \left\|x_{n}-p\right\|^{2}-\left\|\Theta_{n}^{k} x_{n}-\Theta_{n}^{k-1} x_{n}\right\|^{2} .
\end{aligned}
$$

Therefore, by the convexity of $\|\cdot\|^{2}$ and Lemma 2.5 , we get

$$
\begin{aligned}
\left\|y_{n}-p\right\|^{2} & \leq \alpha_{n}\left\|x_{n}-p\right\|^{2}+\left(1-\alpha_{n}\right)\left\|A_{n}^{\lambda_{n}} u_{n}-p\right\|^{2} \\
& \leq \alpha_{n}\left\|x_{n}-p\right\|^{2}+\left(1-\alpha_{n}\right)\left\|u_{n}-p\right\|^{2} \\
& \leq \alpha_{n}\left\|x_{n}-p\right\|^{2}+\left(1-\alpha_{n}\right)\left\|\Theta_{n}^{k} x_{n}-p\right\|^{2} \\
& \leq \alpha_{n}\left\|x_{n}-p\right\|^{2}+\left(1-\alpha_{n}\right)\left(\left\|x_{n}-p\right\|^{2}-\left\|\Theta_{n}^{k} x_{n}-\Theta_{n}^{k-1} x_{n}\right\|^{2}\right) \\
& =\left\|x_{n}-p\right\|^{2}-\left(1-\alpha_{n}\right)\left\|\Theta_{n}^{k} x_{n}-\Theta_{n}^{k-1} x_{n}\right\|^{2} .
\end{aligned}
$$

It follows that

$$
\left(1-\alpha_{n}\right)\left\|\Theta_{n}^{k} x_{n}-\Theta_{n}^{k-1} x_{n}\right\|^{2} \leq\left\|x_{n}-p\right\|^{2}-\left\|y_{n}-p\right\|^{2} \leq\left\|x_{n}-y_{n}\right\|\left(\left\|x_{n}-p\right\|+\left\|y_{n}-p\right\|\right) .
$$

Since $\left\{\alpha_{n}\right\} \subset[0, a]$, we get from (3.12) that (3.13) holds; then we have

$$
\left\|u_{n}-x_{n}\right\| \leq\left\|u_{n}-\Theta_{n}^{M-1} x_{n}\right\|+\left\|\Theta_{n}^{M-1} x_{n}-\Theta_{n}^{M-2} x_{n}\right\|+\cdots+\left\|\Theta_{n}^{1} x_{n}-x_{n}\right\| \rightarrow 0 .
$$


Observe that $\left\|y_{n}-u_{n}\right\| \leq\left\|y_{n}-x_{n}\right\|+\left\|x_{n}-u_{n}\right\|$; we also have $\left\|y_{n}-u_{n}\right\| \rightarrow 0$ as $n \rightarrow \infty$. On the other hand, from $y_{n}=\alpha_{n} x_{n}+\left(1-\alpha_{n}\right) A_{n}^{\lambda_{n}} u_{n}$, we observe that

$$
\begin{aligned}
\left(1-\alpha_{n}\right)\left\|A_{n}^{\lambda_{n}} u_{n}-u_{n}\right\| & =\left\|\left(1-\alpha_{n}\right)\left(A_{n}^{\lambda_{n}} u_{n}-u_{n}\right)\right\| \\
& =\left\|y_{n}-u_{n}-\alpha_{n}\left(x_{n}-u_{n}\right)\right\| \\
& \leq\left\|y_{n}-u_{n}\right\|+\alpha_{n}\left\|x_{n}-u_{n}\right\| .
\end{aligned}
$$

From $\left\{\alpha_{n}\right\} \subset[0, a],(3.19)$, and $\left\|y_{n}-u_{n}\right\| \rightarrow 0$, we obtain $\left\|A_{n}^{\lambda_{n}} u_{n}-u_{n}\right\| \rightarrow 0$ as $n \rightarrow \infty$. It is easy to see that

$$
\left\|A_{n}^{\lambda_{n}} x_{n}-x_{n}\right\| \leq\left\|A_{n}^{\lambda_{n}} x_{n}-A_{n}^{\lambda_{n}} u_{n}\right\|+\left\|A_{n}^{\lambda_{n}} u_{n}-u_{n}\right\|+\left\|u_{n}-x_{n}\right\| \leq 2\left\|u_{n}-x_{n}\right\|+\left\|A_{n}^{\lambda_{n}} u_{n}-u_{n}\right\| .
$$

Combining the above arguments and (3.2), we have

$$
\left\|A_{n}^{\lambda_{n}} x_{n}-x_{n}\right\|=\left\|\lambda_{n} x_{n}+\left(1-\lambda_{n}\right) A_{n} x_{n}-x_{n}\right\|=\left(1-\lambda_{n}\right)\left\|A_{n} x_{n}-x_{n}\right\| .
$$

Now, it follows from $\left\{\lambda_{n}\right\} \subset[\kappa, b]$ that $\left\|A_{n} x_{n}-x_{n}\right\| \rightarrow 0$ as $n \rightarrow \infty$.

Step 5. The following implication holds:

$$
\omega_{w}\left(x_{n}\right) \subset \Omega
$$

We first show that $\omega_{w}\left(x_{n}\right) \subset \cap_{i=1}^{N} F\left(S_{i}\right)$. To this end, we take $\omega \in_{w}\left(x_{n}\right)$ and assume that $x_{n_{j}} \rightarrow \omega$ as $j \rightarrow \infty$ for some subsequence $\left\{x_{n_{j}}\right\}$ of $x_{n}$. Without loss of generality, we may assume that

$$
\eta_{i}^{\left(n_{j}\right)} \rightarrow \eta_{i} \quad(\text { as } j \rightarrow \infty), \quad \forall 1 \leq i \leq N
$$

It is easily seen that each $\eta_{i}>0$ and $\sum_{i=1}^{N} \eta_{i}=1$. We also have

$$
A_{n_{j}} x \rightarrow A x \quad(\text { as } j \rightarrow \infty) \forall x \in C
$$

where $A=\sum_{i=1}^{N} \eta_{i} S_{i}$. Note that, by Proposition 2.4, $A$ is a $\mathcal{\kappa}$-strict pseudocontraction and $F(A)=\cap_{i=1}^{N} F\left(S_{i}\right)$. Since

$$
\begin{aligned}
\left\|A x_{n_{j}}-x_{n_{j}}\right\| & \leq\left\|A_{n_{j}} x_{n_{j}}-A x_{n_{j}}\right\|+\left\|A_{n_{j}} x_{n_{j}}-x_{n_{j}}\right\| \\
& \leq \sum_{i=1}^{N}\left|\eta_{i}^{\left(n_{j}\right)}-\eta_{i}\right|\left\|S_{i} x_{n_{j}}\right\|+\left\|A_{n_{j}} x_{n_{j}}-x_{n_{j}}\right\|,
\end{aligned}
$$


we obtain by virtue of (3.10) and (3.24) that

$$
\lim _{n \rightarrow \infty}\left\|A x_{n_{j}}-x_{n_{j}}\right\|=0
$$

So by the demiclosedness principle (Proposition 2.4 (ii)), it follows that $\omega \in F(A)=\cap_{i=1}^{N} F\left(S_{i}\right)$, and hence $\omega_{w}\left(x_{n}\right) \subset \cap_{i=1}^{N} F\left(S_{i}\right)$.

Next we will show that $\omega \in \cap_{k=1}^{M} \operatorname{EP}\left(F_{k}\right)$. Indeed, by Lemma 2.6, we have that, for each $k=1,2, \ldots, M$,

$$
F_{k}\left(\Theta_{n}^{k} x_{n}, y\right)+\frac{1}{r_{n}}\left\langle y-\Theta_{n}^{k} x_{n}, \Theta_{n}^{k} x_{n}-\Theta_{n}^{k-1} x_{n}\right\rangle \geq 0, \quad \forall y \in C
$$

From (A2), we get

$$
\frac{1}{r_{n}}\left\langle y-\Theta_{n}^{k} x_{n}, \Theta_{n}^{k} x_{n}-\Theta_{n}^{k-1} x_{n}\right\rangle \geq F_{k}\left(y, \Theta_{n}^{k} x_{n}\right), \quad \forall y \in C
$$

Hence,

$$
\left\langle y-\Theta_{n_{j}}^{k} x_{n_{j}} \frac{\Theta_{n_{j}}^{k} x_{n_{j}}-\Theta_{n_{j}}^{k-1} x_{n_{j}}}{r_{n_{j}}}\right\rangle \geq F_{k}\left(y, \Theta_{n_{j}}^{k} x_{n_{j}}\right), \quad \forall y \in C .
$$

From (3.13), we obtain that $\Theta_{n_{j}}^{k} x_{n_{j}} \rightarrow \omega$ as $j \rightarrow \infty$ for each $k=1,2, \ldots, M$ (especially, $u_{n_{j}}=\Theta_{n_{j}}^{M} x_{n_{j}}$ ). Together with (3.13) and (A4) we have, for each $k=1,2, \ldots, M$, that

$$
0 \geq F_{k}(y, w), \quad \forall y \in C
$$

For any $0<t \leq 1$ and $y \in C$, let $y_{t}=t y+(1-t) \omega$. Since $y \in C$ and $\omega \in C$, we obtain that $y_{t} \in C$, and hence $F_{k}\left(y_{t}, \omega\right) \leq 0$. So, we have

$$
0=F_{k}\left(y_{t}, y_{t}\right) \leq t F_{k}\left(y_{t}, y\right)+(1-t) F_{k}\left(y_{t}, \omega\right) \leq t F_{k}\left(y_{t}, y\right)
$$

Dividing by $\mathrm{t}$, we get, for each $k=1,2, \ldots, M$, that

$$
F_{k}\left(y_{t}, y\right) \geq 0, \quad \forall y \in C
$$

Letting $t \rightarrow 0$ and from (A3), we get

$$
F_{k}(\omega, y) \geq 0
$$

for all $y \in C$ and $\omega \in \operatorname{EP}\left(F_{k}\right)$ for each $k=1,2, \ldots, M$, that is, $\omega \in \cap_{k=1}^{M} \operatorname{EP}\left(F_{k}\right)$. Hence (3.23) holds. 
Step 6. From (3.6) and Lemma 2.3, we conclude that $x_{n} \rightarrow q$, where $q=P_{\Omega} x_{1}$.

Remark 3.2. In 2007, Acedo and Xu studied the following CQ method [9]:

$$
\begin{aligned}
x_{0} & \in C \text { chosen arbitrarily, } \\
y_{n} & =\alpha_{n} x_{n}+\left(1-\alpha_{n}\right) A_{n} x_{n}, \\
C_{n} & =\left\{z \in C:\left\|y_{n}-z\right\|^{2} \leq\left\|x_{n}-z\right\|^{2}-\left(1-\alpha_{n}\right)\left(\alpha_{n}-\kappa\right)\left\|x_{n}-A_{n} x_{n}\right\|^{2}\right\}, \\
Q_{n} & =\left\{z \in C:\left\langle x_{n}-z, x_{0}-x_{n}\right\rangle \geq 0\right\}, \\
x_{n+1} & =P_{C_{n} \cap Q_{n}} x_{0} .
\end{aligned}
$$

In this paper, we first turn the strict pseudocontraction $A_{n}$ into nonexpansive mapping $A_{n}^{\lambda_{n}}$ then replace $C_{n}$ with a more simple form in the iterative algorithm.

Remark 3.3. If $F_{k}(x, y)=0, N=1$, and $\lambda_{n}=\delta$, we can obtain [14, Theorem 1].

Remark 3.4. If $M=1, N=1, \kappa=0$, and $\lambda_{n}=0$ and we use $1-\alpha_{n}$ to replace $\alpha_{n}$, we can get the result that has been studied by Tada and Takahashi in [8] for nonexpansive mappings. If $F_{k}(x, y)=0, N=1, \kappa=0$, and $\lambda_{n}=0$, we can get [7, Theorem 3.1].

Theorem 3.5. Let $C$ be a nonempty, closed, and convex subset of a real Hilbert space $H$, and let $F_{k}, k \in\{1,2, \ldots\}$, be bifunctions from $C \times C$ to $\mathbb{R}$ which satisfies conditions (A1)-(A4). Let, for each $1 \leq i \leq N, S_{i}: C \rightarrow C$ be a $\kappa_{i}$-strict pseudocontraction for some $0 \leq \kappa_{i}<1$. Let $\kappa=\max \left\{\kappa_{i}:\right.$ $1 \leq i \leq N\}$. Assume that $\Omega=\cap_{i=1}^{N} F\left(S_{i}\right) \cap\left(\cap_{k=1}^{M} E P\left(F_{k}\right)\right) \neq \emptyset$. Assume also that $\left\{\eta_{i}^{(n)}\right\}_{i=1}^{N}$ is a finite sequence of positive numbers such that $\sum_{i=1}^{N} \eta_{i}^{(n)}=1$ for all $n$ and $\inf _{n \geq 1} \eta_{i}^{(n)}>0$ for all $1 \leq i \leq N$. Let the mapping $A_{n}$ be defined by

$$
A_{n}=\sum_{i=1}^{N} \eta_{i}^{(n)} S_{i} .
$$

Given $x_{1} \in C=C_{1}$, let $x_{n}, u_{n}$, and $y_{n}$ be sequences which are generated by the following algorithm:

$$
\begin{aligned}
u_{n} & =T_{r_{M, n}}^{F_{M}} T_{r_{M-1, n}}^{F_{M-1}} \cdots T_{r_{2, n}}^{F_{2}} T_{r_{1, n}}^{F_{1}} x_{n}, \\
A_{n}^{\lambda_{n}} & =\lambda_{n} I+\left(1-\lambda_{n}\right) A_{n}, \\
y_{n} & =\alpha_{n} x_{n}+\left(1-\alpha_{n}\right) A_{n}^{\lambda_{n}} u_{n}, \\
C_{n+1} & =\left\{z \in C_{n}:\left\|y_{n}-z\right\| \leq\left\|x_{n}-z\right\|\right\}, \\
x_{n+1} & =P_{C_{n+1}} x_{1},
\end{aligned}
$$

where $\left\{\alpha_{n}\right\} \subset[0, a]$ for some $a \in[0,1),\left\{\lambda_{n}\right\} \subset[\kappa, b]$ for some $b \in[\kappa, 1)$, and, $\left\{r_{k, n}\right\} \subset(0, \infty)$ satisfies $\lim _{\inf _{n \rightarrow \infty}} r_{k, n}>0$ for all $k \in\{1,2, \ldots, M\}$. Then, $\left\{x_{n}\right\}$ converge strongly to $P_{\Omega} x_{1}$. 
Proof. The proof of this theorem is similar to that of Theorem 3.1.

Step 1 . The sequence $\left\{x_{n}\right\}$ is well defined. We will show by induction that $C_{n}$ is closed and convex for all $n$. For $n=1$, we have $C=C_{1}$ which is closed and convex. Assume that $C_{n}$ for some $n \geq 1$ is closed and convex; from Lemma 2.2, we have that $C_{n+1}$ is also closed and convex; The proof of $\Omega \subset C_{n}$ is similar to the one in Step 1 of Theorem 3.1.

Step 2. $\left\|x_{n}-x_{1}\right\| \leq\left\|q-x_{1}\right\|$ for all $n$, where $q=P_{\Omega} x_{1}$.

Step 3. $\left\|x_{n+1}-x_{n}\right\| \rightarrow 0$ as $n \rightarrow \infty$.

Step $4 .\left\|A_{n} x_{n}-x_{n}\right\| \rightarrow 0$ as $n \rightarrow \infty$.

Step 5. $\omega_{w}\left(x_{n}\right) \subset \Omega$.

Step 6. $x_{n} \rightarrow q$.

The proof of Step 2-Step 6 is similar to that of Theorem 3.1.

Remark 3.6. If $M=1$, we can obtain the two corresponding theorems in [10].

\section{Cyclic Algorithm}

Let $C$ be a closed, and convex subset of a Hilbert space $H$, and let $\left\{S_{i}\right\}_{i=0}^{N-1}$ be $N \kappa_{i}$-strict pseudocontractions on $C$ such that the common fixed point set

$$
\bigcap_{i=0}^{N-1} F\left(S_{i}\right) \neq \emptyset
$$

Let $x_{0} \in C$, and let $\left\{\alpha_{n}\right\}_{n=0}^{\infty}$ be a sequence in $(0,1)$. The cyclic algorithm generates a sequence $\left\{x_{n}\right\}_{n=1}^{\infty}$ in the following way:

$$
\begin{aligned}
x_{1} & =\alpha_{0} x_{0}+\left(1-\alpha_{0}\right) S_{0} x_{0}, \\
x_{2} & =\alpha_{1} x_{1}+\left(1-\alpha_{1}\right) S_{1} x_{1} \\
\ldots & \\
x_{N} & =\alpha_{N-1} x_{N-1}+\left(1-\alpha_{N-1}\right) S_{N-1} x_{N-1}, \\
x_{N+1} & =\alpha_{N} x_{N}+\left(1-\alpha_{N}\right) S_{0} x_{N},
\end{aligned}
$$

In general, $x_{n+1}$ is defined by

$$
x_{n+1}=\alpha_{n} x_{n}+\left(1-\alpha_{n}\right) S_{[n]} x_{n},
$$

where $S_{[n]}=S_{i}$, with $i=n(\bmod ) N, 0 \leq i \leq N-1$. 
Theorem 4.1. Let $C$ be a nonempty, closed, and convex subset of a real Hilbert space $H$, and let $F_{k}, k \in\{1,2, \ldots, M\}$, be bifunctions from $C \times C$ to $\mathbb{R}$ which satisfies conditions (A1)-(A4). Let, for each $0 \leq i \leq N-1, S_{i}: C \rightarrow C$ be a $\kappa_{i}$-strict pseudocontraction for some $0 \leq \kappa_{i}<1$. Let $\mathcal{\kappa}=\max \left\{\kappa_{i}: 0 \leq i \leq N-1\right\}$. Assume that $\Omega=\cap_{i=0}^{N-1} F\left(S_{i}\right) \cap\left(\cap_{k=1}^{M} \operatorname{EP}\left(F_{k}\right)\right) \neq \emptyset$. Given $x_{0} \in C$, let $x_{n}, u_{n}$, and $y_{n}$ be sequences which are generated by the following algorithm:

$$
\begin{aligned}
u_{n} & =T_{r_{M, n}}^{F_{M}} T_{r_{M-1, n}}^{F_{M-1}} \cdots T_{r_{2, n}}^{F_{2}} T_{r_{1, n}}^{F_{1}} x_{n}, \\
S_{[n]}^{\lambda_{n}} & =\lambda_{n} I+\left(1-\lambda_{n}\right) S_{[n]}, \\
y_{n} & =\alpha_{n} x_{n}+\left(1-\alpha_{n}\right) S_{[n]}^{\lambda_{n}} u_{n}, \\
C_{n} & =\left\{z \in C:\left\|y_{n}-z\right\| \leq\left\|x_{n}-z\right\|\right\}, \\
Q_{n} & =\left\{z \in C:\left\langle x_{n}-z, x_{0}-x_{n}\right\rangle \geq 0\right\}, \\
x_{n+1} & =P_{C_{n} \cap Q_{n}} x_{0},
\end{aligned}
$$

where $\left\{\alpha_{n}\right\} \subset[0, a]$ for some $a \in[0,1),\left\{\lambda_{n}\right\} \subset[\kappa, b]$ for some $b \in[\kappa, 1)$, and $\left\{r_{k, n}\right\} \subset(0, \infty)$ satisfies $\liminf _{n \rightarrow \infty} r_{k, n}>0$ for all $k \in\{1,2, \ldots, M\}$. Then, $\left\{x_{n}\right\}$ converge strongly to $P_{F} x_{0}$.

Proof. The proof of this theorem is similar to that of Theorem 3.1. The main points are the following.

Step 1 . The sequence $\left\{x_{n}\right\}$ is well defined.

Step 2. $\left\|x_{n}-x_{0}\right\| \leq\left\|q-x_{0}\right\|$ for all $n$, where $q=P_{\Omega} x_{0}$.

Step 3. $\left\|x_{n+1}-x_{n}\right\| \rightarrow 0$.

Step 4. $\left\|S_{[n]} x_{n}-x_{n}\right\| \rightarrow 0$. To prove the above steps, one simply replaces $A_{n}$ with $S_{[n]}$ in the proof of Theorem 3.1.

Step 5. $\omega_{w}\left(x_{n}\right) \subset \Omega$. Indeed, let $\omega \in \omega_{w}\left(x_{n}\right)$ and $x_{n_{m}} \rightarrow \omega$ for some subsequence $\left\{x_{n_{m}}\right\}$ of $\left\{x_{n}\right\}$. We may assume that $l=n_{m}(\bmod N)$ for all $m$. Since, by $\left\|x_{n+1}-x_{n}\right\| \rightarrow 0$, we also have $x_{n_{m}+j} \rightarrow \omega$ for all $j \geq 0$, we deduce that

$$
\left\|x_{n_{m}+j}-S_{[l+j]} x_{n_{m}+j}\right\|=\left\|x_{n_{m}+j}-S_{\left[n_{m}+j\right]} x_{n_{m}+j}\right\| \rightarrow 0 .
$$

Then the demiclosedness principle implies that $\omega \in F\left(S_{[l+j]}\right)$ for all $j$. This ensures that $\omega \in$ $\cap_{i=1}^{N} F\left(S_{i}\right)$.The Proof of $\omega \in \cap_{k=1}^{M} \mathrm{EP}\left(F_{k}\right)$ is similar to that of Theorem 3.1.

Step 6 . The sequence $x_{n}$ converges strongly to $q$.

The strong convergence to $q$ of $\left\{x_{n}\right\}$ is a consequence of Step 2, Step 5, and Lemma 2.3.

Theorem 4.2. Let $C$ be a nonempty, closed, and convex subset of a real Hilbert space $H$, and let $F_{k}, k \in\{1,2, \ldots, M\}$, be bifunctions from $C \times C$ to $\mathbb{R}$ which satisfies conditions (A1)-(A4). Let, for each $0 \leq i \leq N-1, S_{i}: C \rightarrow C$ be a $\kappa_{i}$-strict pseudocontraction for some $0 \leq \kappa_{i}<1$. Let 
$\kappa=\max \left\{\kappa_{i}: 0 \leq i \leq N-1\right\}$. Assume that $\Omega=\cap_{i=0}^{N-1} F\left(S_{i}\right) \cap\left(\cap_{k=1}^{M} \operatorname{EP}\left(F_{k}\right)\right) \neq \emptyset$. Given $x_{0} \in C=C_{0}$, let $x_{n}, u_{n}$, and $y_{n}$ be sequences whic are generated by the following algorithm:

$$
\begin{aligned}
u_{n} & =T_{r_{M, n}}^{F_{M}} T_{r_{M-1, n}}^{F_{M-1}} \cdots T_{r_{2, n}}^{F_{2}} T_{r_{1, n}}^{F_{1}} x_{n}, \\
S_{[n]}^{\lambda_{n}} & =\lambda_{n} I+\left(1-\lambda_{n}\right) S_{[n]}, \\
y_{n} & =\alpha_{n} x_{n}+\left(1-\alpha_{n}\right) S_{[n]}^{\lambda_{n}} u_{n}, \\
C_{n+1} & =\left\{z \in C_{n}:\left\|y_{n}-z\right\| \leq\left\|x_{n}-z\right\|\right\}, \\
x_{n+1} & =P_{C_{n+1}} x_{0},
\end{aligned}
$$

where $\left\{\alpha_{n}\right\} \subset[0, a]$ for some $a \in[0,1),\left\{\lambda_{n}\right\} \subset[\kappa, b]$ for some $b \in[\kappa, 1)$, and $\left\{r_{k, n}\right\} \subset(0, \infty)$ satisfies $\lim _{\inf _{n \rightarrow \infty}} r_{k, n}>0$ for all $k \in\{1,2, \ldots\}$. Then, $\left\{x_{n}\right\}$ converge strongly to $P_{\Omega} x_{0}$.

Proof. The proof of this theorem is similar to that of Step 1 in Theorem 3.5 and Step 2-Step 6 in Theorem 4.1.

Remark 4.3. If $M=1$, we can obtain the two corresponding theorems in [10].

\section{Acknowledgment}

This research is supported by Fundamental Research Funds for the Central Universities (GRANT:ZXH2009D021).

\section{References}

[1] P. L. Combettes and S. A. Hirstoaga, "Equilibrium programming in Hilbert spaces," Journal of Nonlinear and Convex Analysis, vol. 6, no. 1, pp. 117-136, 2005.

[2] F. E. Browder and W. V. Petryshyn, "Construction of fixed points of nonlinear mappings in Hilbert space," Journal of Mathematical Analysis and Applications, vol. 20, pp. 197-228, 1967.

[3] E. Blum and W. Oettli, "From optimization and variational inequalities to equilibrium problems," The Mathematics Student, vol. 63, no. 1-4, pp. 123-145, 1994.

[4] V. Colao, G. Marino, and H. K. Xu, "An iterative method for finding common solutions of equilibrium and fixed point problems," Journal of Mathematical Analysis and Applications, vol. 344, no. 1, pp. 340352, 2008.

[5] Y. Liu, "A general iterative method for equilibrium problems and strict pseudo-contractions in Hilbert spaces," Nonlinear Analysis. Theory, Methods E Applications, vol. 71, no. 10, pp. 4852-4861, 2009.

[6] G. Marino and H. K. Xu, "Weak and strong convergence theorems for strict pseudo-contractions in Hilbert spaces," Journal of Mathematical Analysis and Applications, vol. 329, no. 1, pp. 336-346, 2007.

[7] K. Nakajo and W. Takahashi, "Strong convergence theorems for nonexpansive mappings and nonexpansive semigroups," Journal of Mathematical Analysis and Applications, vol. 279, no. 2, pp. 372 $379,2003$.

[8] A. Tada and W. Takahashi, "Weak and strong convergence theorems for a nonexpansive mapping and an equilibrium problem," Journal of Optimization Theory and Applications, vol. 133, no. 3, pp. 359-370, 2007.

[9] G. L. Acedo and H. K. Xu, "Iterative methods for strict pseudo-contractions in Hilbert spaces," Nonlinear Analysis. Theory, Methods \& Applications, vol. 67, no. 7, pp. 2258-2271, 2007.

[10] P. C. Duan and J. Zhao, "Strong convergence theorems by hybrid methods for strict pseudocontractions and equilibrium problems," Fixed Point Theory and Applications, vol. 2010, Article ID 528307, 13 pages, 2010. 
[11] P. Kumam, "A hybrid approximation method for equilibrium and fixed point problems for a monotone mapping and a nonexpansive mapping," Nonlinear Analysis. Hybrid Systems, vol. 2, no. 4, pp. 1245-1255, 2008.

[12] W. Takahashi, Y. Takeuchi, and R. Kubota, "Strong convergence theorems by hybrid methods for families of nonexpansive mappings in Hilbert spaces," Journal of Mathematical Analysis and Applications, vol. 341, no. 1, pp. 276-286, 2008.

[13] C. Martinez-Yanes and H. K. Xu, "Strong convergence of the CQ method for fixed point iteration processes," Nonlinear Analysis. Theory, Methods E Applications, vol. 64, no. 11, pp. 2400-2411, 2006.

[14] Y. H. Yao and R. D. Chen, "Strong convergence theorems for strict pseudo-contractions in Hilbert spaces," Journal of Applied Mathematics and Computing, vol. 32, no. 1, pp. 69-82, 2010. 